\title{
辅酶 $\mathbf{B}_{12}$ 模型化合物的研究 *
}

VII. $\mathrm{C}_{2} \mathrm{H}_{5} \mathrm{Co}(\mathrm{SB})$ 和 $\mathrm{RCo}(\mathrm{salen}) \mathrm{L}$ 系列 化合物的 CNDO/2 计算

\author{
韩德艳 $^{1)}$ 缪 强 俞马宏 ${ }^{2)}$ 倪 浩 陈慧兰 ${ }^{3}$ 唐雯霞
}

(南京大学化学系, 配位化学国家重点实验室, 南京 210008)

\section{关键词辅酸 $\mathrm{B}_{12}$ 模型化合物、Co-C 键、键级、CNDO/2}

目前合成系列结构上呈规律性变化的辅酶 $\mathrm{B}_{12}$ 模型化合物和研究其结构与 $\mathrm{Co}-\mathrm{C}$ 键 性质间的关系, 是认识影响辅酶 $B_{12}$ 中 $\mathrm{Co}-\mathrm{C}$ 键稳定性的因素及辅酶的催化作用机制的有效 途径 ${ }^{1 !}$. 而有关模型化合物的微观反应机制的研究还很少, Lipscomb 等人用 PRDDO 方法 计算了简单模型体系 $\mathrm{CH}_{3}-\mathrm{Co}\left(\mathrm{NH}_{3}\right)_{5}^{[2]}$; 朱龙根等人用非参数自洽场方法计算了模型化合物 的各种构型变化对 $\mathrm{Co}-\mathrm{C}$ 键强度的影响 ${ }^{[3]}$, Mealli 等人用定量分子轨道方法和微扰理论讨论 了 $\mathrm{Co}-\mathrm{C}$ 键的离解过程 ${ }^{[4]}$, 但真正根据模型化合物的晶体结构数据进行量化计算还很少见. 我们合成并表征了两类西夫碱烷基钴配合物, 它们的平面配体分别为 salen (双水杨酫合乙 二亚胺 ) 和 $\mathrm{SB}$ (双水杨醛合 $2^{\prime}, 3^{\prime}$-二甲基 $-2^{\prime}, 3^{\prime}$ - 丁二胺), 其结构如下:

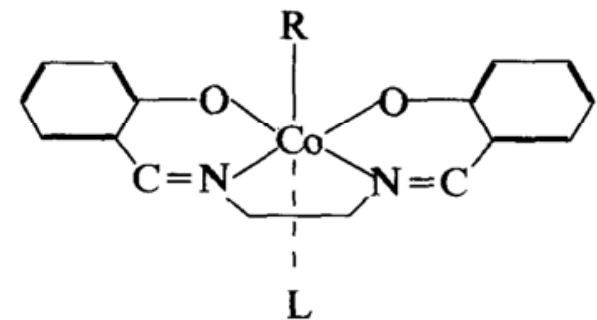

RCo (salen) L

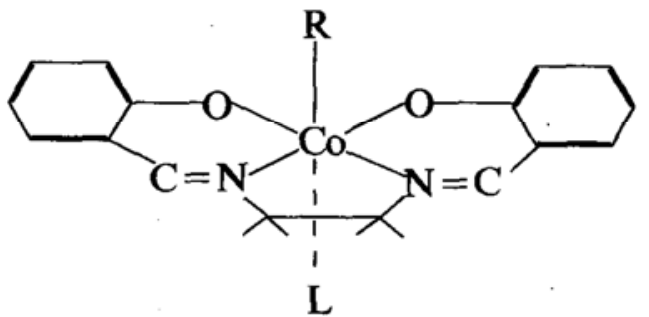

RCo (SB) L

研究了模型化合物的固态和溶液中 $\mathrm{Co}-\mathrm{C}$ 键的稳定性 ${ }^{[5, \text {, }}$, 培养了 6 个单晶并进行了结构 分析, 它们是 $\mathrm{i}-\mathrm{C}_{4} \mathrm{H}_{9} \mathrm{Co}$ (salen) $\mathrm{H}_{2} \mathrm{O}$ (1)、i- $\mathrm{C}_{4} \mathrm{H}_{9} \mathrm{Co}$ (salen) $\mathrm{py}^{\text {[7] }}$ (2), $\mathrm{i}-\mathrm{C}_{4} \mathrm{H}_{9} \mathrm{Co}$ (salen) ( $\gamma$-pic) (3), $\mathrm{C}_{3} \mathrm{H}_{7} \mathrm{Co}$ (salen) ( $\gamma$-pic) (4), $\mathrm{C}_{6} \mathrm{H}_{5} \mathrm{C}_{2} \mathrm{H}_{4} \mathrm{Co}$ (salen) $\mathrm{H}_{2} \mathrm{O}$ (5), $\mathrm{C}_{2} \mathrm{H}_{5} \mathrm{Co}$ (SB) (6). 本工作用半经验分 子轨道理论中的全略微分重叠理论法对 $\mathrm{C}_{2} \mathrm{H}_{5} \mathrm{Co}(\mathrm{SB})$ 和 RCo (salen) L 系列辅酶 $\mathrm{B}_{12}$ 模型化 合物进行计算, 得出了有意义的结果.

\section{1 计算方法和数据}

计算采用 $\mathrm{CNDO} / 2$ 法, 其参数取自文献[8], 计算依据的部分键长、键角数据列于表 1 .

1993-07-28 收稿, 1993-10-08 收修改稿.

*国家自然科学基金资助项目.

1) 现在工作单位: 湖北师范学院化学系. 2) 工作单位: 南京理工大学化学化工学院. 3) 联系人. 
表 1 重要的键长、键角数据 ${ }^{\mathrm{a})}$

\begin{tabular}{|c|c|c|c|c|c|c|c|}
\hline & 化 合 物 & 1 & $2^{17}$ & 3 & 4 & 5 & 6 \\
\hline \multirow{3}{*}{ 键 } & $\mathrm{Co}-\mathrm{C} 5$ & $1.991(9)$ & $2.003(4)$ & $2.027(6)$ & $2.008(6)$ & $1.962(14)$ & $2.018(7)$ \\
\hline & $\mathrm{Co}-\mathrm{O} 1$ & $1.892(7)$ & $1.908(2)$ & $1.896(3)$ & $1.907(4)$ & $1.903(10)$ & $1.870(3)$ \\
\hline & $\mathrm{Co}-\mathrm{O} 2$ & $1.911(6)$ & $1.901(2)$ & $1.903(3)$ & $1.892(5)$ & $1.899(10)$ & $1.875(4)$ \\
\hline 长 & $\mathrm{Co}-\mathrm{O} 3$ & $2.218(6)$ & & & & $2.209(9)$ & \\
\hline \multirow{3}{*}{$(\dot{\mathrm{A}})$} & $\mathrm{Co}-\mathrm{N} 1$ & $1.861(8)$ & $1.881(3)$ & $1.873(4)$ & $1.880(5)$ & $1.868(11)$ & $1.875(4)$ \\
\hline & $\mathrm{Co}-\mathrm{N} 2$ & $1.859(8)$ & $1.878(3)$ & $1.867(5)$ & $1.869(5)$ & $1.842(13)$ & $1.885(4)$ \\
\hline & $\mathrm{Co}-\mathrm{N} 3$ & & $2.184(3)$ & $2.230(4)$ & $2.179(4)$ & & \\
\hline \multirow{3}{*}{ 键 } & $\mathrm{Co}-\mathrm{C} 5-\mathrm{C}$ & $126.98(8)$ & $115.2(3)$ & $123.7(9)$ & $116.7(3)$ & $119.0(1)$ & $113.9(4)$ \\
\hline & $\mathrm{C} 5-\mathrm{Co}-\mathrm{O} 1$ & $91.9(4)$ & $92.6(1)$ & $90.4(2)$ & $94.0(2)$ & $91.7(5)$ & $94.6(2)$ \\
\hline & $\mathrm{C} 5-\mathrm{Co}-\mathrm{O} 2$ & $88.3(4)$ & $87.6(1)$ & $86.8(2)$ & $89.5(2)$ & $89.9(5)$ & $93.9(2)$ \\
\hline 角 & $\mathrm{C} 5-\mathrm{Co}-\mathrm{O} 3$ & $175.9(4)$ & & & & $176.6(5)$ & \\
\hline \multirow{3}{*}{$\left({ }^{\circ}\right)$} & $\mathrm{C} 5-\mathrm{Co}-\mathrm{N} 1$ & $97.3(4)$ & $91.8(2)$ & $92.1(3)$ & $89.7(3)$ & $95.2(5)$ & $96.0(2)$ \\
\hline & $\mathrm{C} 5-\mathrm{Co}-\mathrm{N} 2$ & $92.1(4)$ & $91.5(1)$ & $92.1(2)$ & $90.0(2)$ & $92.8(6)$ & $96.7(2)$ \\
\hline & $\mathrm{C} 5-\mathrm{Co}-\mathrm{N} 3$ & & $175.3(2)$ & $175.5(2)$ & $176.9(2)$ & & \\
\hline
\end{tabular}

a) C5 为轴向烷基与 Co 相连的原子, O1O2NIN2 为平面配位原子, N3 为轴向践基的配位原子.

\section{2 计算结果与讨论}

模型化合物的部分计算结果如表 2. 对 i- $\mathrm{C}_{4} \mathrm{H}_{9} \mathrm{Co}$ (salen) L 系列模型化合物 (1), (2), (3),

表 2 模型化合物的部分计算结果

\begin{tabular}{|c|c|c|c|c|c|c|}
\hline 配 合 物 & 1 & 2 & 3 & 4 & 5 & 6 \\
\hline 电子能量 (a.u.) & -1659.979 & -2088.203 & $-2167,208$ & -2018.264 & -1915.483 & -1717.738 \\
\hline 总能量 (a.u.) & -259.095 & -289.224 & -299.039 & -288.265 & -287.645 & -256.531 \\
\hline $\mathrm{Co}-\mathrm{C}$ 键级 & 1.0017 & 0.9599 & 0.9423 & 0.9732 & 1.0309 & 0.9334 \\
\hline $\mathrm{Co}--\mathrm{C}$ 重香集居 (e) & 0.8177 & 0.7908 & 0.7709 & 0.8006 & 0.8569 & 0.8177 \\
\hline$E_{\text {HUMO }}$ (a.u.) & -0.1945 & -0.1676 & -0.1764 & -0.1744 & -0.1981 & -0.1911 \\
\hline$E_{\text {LUMO }}$ (a.u.) & 0.1037 & -0.0028 & 0.0035 & 0.0008 & 0.0015 & 0.0626 \\
\hline 原 $\quad \mathrm{Co}$ & 1.5904 & 1.5865 & 1.5813 & 1.5708 & 1.5267 & 1.4638 \\
\hline $\mathrm{C}$ & -0.3762 & -0.3878 & -0.3731 & -0.3171 & -0.3105 & -0.2823 \\
\hline 01 & -0.3785 & -0.3936 & -0.3919 & -0.3847 & -0.3902 & -0.3296 \\
\hline $\mathrm{O} 2$ & -0.3944 & -0.3977 & -0.3999 & -0.3952 & -0.4171 & -0.3307 \\
\hline $\mathrm{N} 1$ & -0.2336 & -0.2318 & -0.2348 & -0.2518 & -0.1992 & -0.2202 \\
\hline $\mathrm{N} 2$ & -0.2207 & -0.2498 & -0.2464 & -0.2601 & -0.1876 & -0.2466 \\
\hline (e) & -0.2724 & -0.1422 & -0.1599 & -0.1481 & -0.3061 & \\
\hline
\end{tabular}

当 $\mathrm{L}$ 为 $\mathrm{H}_{2} \mathrm{O} \rightarrow$ py 或 $\gamma$-pic 时, 其前线最高占有轨道能级 $\mathrm{E}_{\text {номо 由 }}-0.1945$ 升为 -0.1676 或 - 0.1764a.u., 这表明吡啶 (或甲基吡啶) 配位后, 模型化合物易与其它外来亲电试剂发生 氧化反应; 相反, 其最低空轨道能级 $E_{\mathrm{LUMO}}$ 由 0.1037 降为 -0.0028 或 $0.0035 \mathrm{a}$. u., 因此轴 向有机碱配位后, 增大了模型配合物容纳电子的能力. 对于六配位的化合物 (1)-(5), 计算所 得 $\mathrm{Co}-\mathrm{C}$ 键级与它们键长的变化是一致的, 即随着 $\mathrm{Co}-\mathrm{C}$ 键长的增加, 键级减小. 表 2 中五 配位化合物的 $\mathrm{Co}-\mathrm{C}$ 键级最低, 虽然它的 $\mathrm{Co}-\mathrm{C}$ 键长与其它化合物 (除 5) 差别不大, 该结果 
与其平面配体具有较大位阻导致 $\mathrm{Co}-\mathrm{C}$ 键减弱有关. TG-DSC 实验测得的失烷基温度也是 $\mathrm{C}_{2} \mathrm{H}_{5} \mathrm{Co}$ (SB) $<\mathrm{C}_{2} \mathrm{H}_{5} \mathrm{Co}$ (salen) . 另外, 中心金属钴配位的原子上的电荷分布计算表明：轴向加 了吡啶或甲基吡啶类有机碱后, 部分电荷发生转移, 一部分流向 $\mathrm{Co}$, 一部分流向平面配体, 使 Co 的正电荷略有降低, 而 $\mathrm{O} 1 \mathrm{O} 2 \mathrm{~N} 1 \mathrm{~N} 2$ 负电荷略升高.

对于 $\mathrm{RCO}$ (salen) $\mathrm{H}_{2} \mathrm{O}\left(\mathrm{R}=\mathrm{CH}_{3}, \mathrm{C}_{2} \mathrm{H}_{5}, \mathrm{i}-\mathrm{C}_{3} \mathrm{H}_{7}, \mathrm{n}-\mathrm{C}_{3} \mathrm{H}_{7}, \mathrm{n}-\mathrm{C}_{4} \mathrm{H}_{9}\right)$ 系列化合物也进行了 $\mathrm{CNDO} / 2$ 计算, 以 $\mathrm{i}-\mathrm{C}_{4} \mathrm{H}_{9} \mathrm{Co}$ (salen) $\mathrm{H}_{2} \mathrm{O}$ 的晶体结构数据为依据, 我们通过改变 $\mathrm{Co}-\mathrm{C} 5-\mathrm{C}(\mathrm{R})$ 角 $\left(109.5-126^{\circ}\right)$ 和 $\mathrm{N} 1-\mathrm{Co}-\mathrm{C} 5$ 角 $\left(87-107^{\circ}\right)$ (根据前人的计算结果, 上述两个角度的变化对 总能量和 $\mathrm{Co}-\mathrm{C}$ 键级的影响比改变键长影响大得多 ${ }^{[3]}$ ) 计算结构变化最大的甲基化合物的总能 量和 $\mathrm{Co}-\mathrm{C} 5$ 键级, 发现取 $\mathrm{i}-\mathrm{C}_{4} \mathrm{H}_{9} \mathrm{Co}$ (salen) $\mathrm{H}_{2} \mathrm{O}$ 的晶体结构数据时化合物总能量最低和 $\mathrm{Co}-\mathrm{C} 5$ 键级最大, 对其它的化合物也进行了类似计算, 部分计算结果列于表 3.

表 $3 \mathrm{RCo}$ (salen) $\mathrm{H}_{2} \mathrm{O}$ 系列配合物部分计算结果

\begin{tabular}{|c|c|c|c|c|c|}
\hline 配 合 物 (R) & $\mathrm{CH}_{3}$ & $\mathrm{C}_{2} \mathrm{H}_{5}$ & $\mathrm{i}-\mathrm{C}_{3} \mathrm{H}_{7}$ & $\mathrm{n}-\mathrm{C}_{3} \mathrm{H}_{7}$ & $\mathrm{n}-\mathrm{C}_{4} \mathrm{H}_{9}$ \\
\hline 电子能量 (a.u.) & -1345.090 & -1449.159 & -1567.836 & -1545.439 & -1637.452 \\
\hline 总能量 (a.u.) & -223.27 & -241.889 & -250.544 & -250.490 & -259.096 \\
\hline $\mathrm{Co}-\mathrm{C}$ 键级 & 1.0202 & 0.9977 & 0.9482 & 1.0026 & 1.0045 \\
\hline $\mathrm{Co}$ - $\mathrm{C}$ 重叠集居 & 0.8265 & 0.8174 & 0.7952 & 0.8182 & 0.8188 \\
\hline $\begin{array}{ll}\text { 原子上 } & \mathrm{Co}(\mathrm{e}) \\
\text { 净电荷 } & \mathrm{C}(\mathrm{e})\end{array}$ & $\begin{array}{r}1.5795 \\
-0.4401\end{array}$ & $\begin{array}{r}1.5849 \\
-0.3560\end{array}$ & $\begin{array}{r}1.6253 \\
-0.3074\end{array}$ & $\begin{array}{r}1.5909 \\
-0.3723\end{array}$ & $\begin{array}{r}1.5914 \\
-0.3769\end{array}$ \\
\hline
\end{tabular}

由表2、表3 中得出, $\mathrm{RCo}$ (salen) $\mathrm{H}_{2} \mathrm{O}$ 系列 $\mathrm{Co}-\mathrm{C}$ 键级变化顺序为: $\mathrm{C}_{6} \mathrm{H}_{5} \mathrm{C}_{2} \mathrm{H}_{4}>\mathrm{CH}_{3}>\mathrm{n}-\mathrm{C}_{4} \mathrm{H}_{9}$ $\approx \mathrm{i}-\mathrm{C}_{4} \mathrm{H}_{9} \approx \mathrm{n}-\mathrm{C}_{3} \mathrm{H}_{7}>\mathrm{C}_{2} \mathrm{H}_{5}>\mathrm{i}-\mathrm{C}_{3} \mathrm{H}_{7}$, 该顺序反映了 $\mathrm{Co}-\mathrm{C}$ 键的稳定性受 $\mathrm{R}$ 基空间位阻和电子 结构的影响; $\mathrm{i}-\mathrm{C}_{3} \mathrm{H}_{7}$ 化合物键级最小, 说明它的 $\mathrm{Co}-\mathrm{C}$ 键最弱, 这主要是由 $\mathrm{i}-\mathrm{C}_{3} \mathrm{H}_{7}$ 的空间位 阻大造成的; 而 $\mathrm{C}_{6} \mathrm{H}_{5} \mathrm{C}_{2} \mathrm{H}_{4}$ 化合物键级最大, 说明 $\mathrm{Co}-\mathrm{C}$ 键最稳定, 其原因是 $\mathrm{R}$ 基上带有共轭体

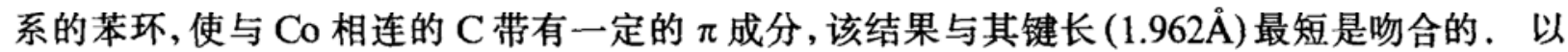
上键级的变化顺序与固态时 $\mathrm{Co}-\mathrm{C}$ 键的断裂温度 (DSC 峰温) 基本一致 ${ }^{[5]}$.

致谢计算过程中曾得到南京理工大学化学化工学院肖鹤鸣教授的指导和帮助, 在此 表示感谢.

\section{参考 文 献}

[1] Dolphin, D. (ed.), B12, Wiley, New York, 1982, 1:2.

[2] Christianson, D. W., Lipscomb, W. N., J. Am. Chem. Soc., 1985, 107:2682.

[ 3] Zhu, L. G., Kostic, N. M., Inorg. Chem., 1987, 26:4194.

[4] Mealli, C., Sabat, M., Marzilli, L. G., J. Am. Chem. Soc., 1987, 109: 1593.

[ 5] 陈慧兰、陈冠萻、唐雯得等, 无机化学学报, 1991, 7(4): 373.

[6] 陈慧兰、陈冠蓄、唐雯霞等, 高等学校化学学报, 1991, 12(4): 431.

[ 7] Chen, H., Han, D., Yon, H. et al., Ployhedron, 1993, 12(9): 1097.

[8] Pople, J. A., Beveridge, D. L., Approximate Molecular Orbital Theory, 1970. 